\title{
003 PRE-CLINICAL EVALUATION OF THE AQUEOUS EXTRACT OF SESBANIA GRANDIFLORA (KATURAY) AS HYPOGLYCEMIC AGENT
}

Jean Nunez Guillasper, John Micah Manuel Gabriel, Aubrey Marie Shalimar Dulay Razalan. Nursing, Nueva Ecija University of Science and Technology, Cabanatuan City, Philippines

10.1136/bmjopen-2015-forum2015abstracts.3

Background Diabetes mellitus (DM) is a chronic multisystem disease related to abnormal insulin production, impaired insulin utilization, or both. Despite of the progress in synthetic chemistry and biotechnology, plants are still an indispensable source of medicinal preparations both preventive and curative. In the Philippines, over 1,020 medicinal plants were documented and ten among those were recommended by the DOH, unfortunately, Sesbaniagrandiflora (L.) Pers (Katuray) is not included among the ten medicinal plants recommended. Further, there is no specific basis that will justify the medicinal use of Sesbaniagrandiflora (L.) Persflowers in the treatment of diabetes. Objectives This study aimed to investigate the hypoglycemic activity of the Aqueous Extract of Sesbaniagrandiflora(L.) Pers (Katuray) flowers among nonhuman primates (Philippine Macaque/MacacafascicularisPhilippinensis) which is said to have a close genetic relationship to human.

Methods The researchers utilized the true experimental research. Four MacacafascicularisPhilippinensis served as the subjects of the study. The test animals were induced with Alloxan Monohydrate that causes hyperglycemia. Baseline data of RBS, FBS and HbA1c was taken prior to the induction of Alloxan Monohydrate. Blood glucose monitoring was done for the period of 1 week.Administration of Insulin and tea fractionate (Aqueous Extract) of SesbaniaGrandiflora(L.) Pers(Katuray) flowers were done on the 4th day where the highest RBS was noted. Blood samples were obtained from the saphenous and cephalic veins of the non-anesthetized subjects. The blood samples were sent to the laboratory for analysis and interpretation. The data were clinically interpreted based on the normal values of the laboratory tests conducted. 
Result The study revealed that the first and second phase of the toxicity test of the tea fractionate (Aqueous extract) of SesbaniaGrandiflora (L.) Pers(Katuray) flowers was found to be non-toxic for the test animals (albino mice) as evidenced by zero mortality rate, however, hyperactivity among the test animals were noted during the second day of observation. The tea fractionate (Aqueous extract) of SesbaniaGrandiflora (L.) Pers (Katuray) flowers and Insulin have similar effects on the blood glucose of the test animals which can reduce almost $90-95 \%$ of the blood glucose levels of diabetic -induced monkeys.

Conclusion The results of this study served as the basis for formulating a potent hypoglycemic drug that can cure Type 2 Diabetes Mellitus and can compete to those synthetic drugsavailable in the market. 